ONCOGENESIS

\title{
Switching off death
}

Since JUN was discovered, almost 20 years ago, it has been shown to regulate proliferation and survival in both normal tissues and tumours. It seems to be particularly important for hepatocytes, which has led to the hypothesis that it is also involved in the development of hepatic carcinomas. Erwin Wagner and colleagues have investigated this in mice, and show that Jun promotes tumorigenesis by switching off p53, which, in turn, switches off death.

A conditional allele system was used to specifically delete Jun in hepatocytes initially at around birth, and the mice were then subjected to a chemical carcinogenesis protocol to induce liver tumours. The control mice had large tumours by the age of 6 months and $60 \%$ had died due to liver failure by 18 months. The liverspecific Jun $\Delta$ mice, on the other hand, had fewer and smaller tumours, and only $10 \%$ had died by 18 months, indicating that the deletion of Jun can suppress liver tumour formation. But does this suppression occur because of decreased proliferation or increased apoptosis?

The authors determined the rate of proliferation and apoptosis in control and Jun $\Delta$ tumours using cellular markers for each - Ki67 and TUNEL, respectively - to investigate this. Although the number of proliferating cells was similar in both tumour types, the number of apoptotic cells was greatly increased in Juns tumours, indicating that Jun acts as a survival factor in liver tumour cells.

To further analyse the requirement for this survival function of Jun, the authors deleted it at different stages of hepatic tumour development. Tumour formation was repressed to a similar extent regardless of whether Jun was deleted before or after tumour initiation, and the survival function also seems to be independent of tumour promotion. The activating amino-terminal phosphorylation of Jun was also not required for this activity, as mutation of these sites to alanine did not impair tumour formation. Interestingly, deleting Jun 6 months after tumour initiation did not then cause tumour regression - the tumours had become independent of Jun. Jun therefore facilitates tumour development by inhibiting apoptosis at an early stage, between initiation and progression.

So how does Jun activity repress apoptosis? The expression levels of several Bcl2-family members and death receptors were not altered in Juns hepatocytes compared with control hepatocytes, but the response to the TNF- $\alpha$ death ligand was. Deletion of Jun seems to sensitize hepatocytes to TNF- $\alpha$-induced apoptosis by increasing the activity of the JNK and p38 stress kinases. These kinases have been shown to activate $\mathrm{p} 53$, which indicates a mechanism for the increased apoptosis in Juns tumours. Indeed, hepatocytes that were deficient for both Jun and $\operatorname{Trp} 53$ had a restored resistance to TNF $\alpha$-induced apoptosis.

But is the same true in tumours? Biopsies from both control and Jun $\Delta$ liver tumours confirmed that p53 levels were increased in the absence of Jun, and that this corresponded with an increase in expression of a pro-apoptotic target of p53, Noxa.

So, in hepatic tumour development, Jun seems to promote tumorigenesis by antagonizing the pro-apoptotic function of $\mathrm{p} 53$. The design of Jun inhibitors might therefore prove a useful therapeutic strategy for this cancer type. Emma Greenwood

(2) References and links ORIGINAL RESEARCH PAPER Eferl, $R$. et al. Liver tumor development: c-Jun antagonizes the proapoptotic activity of p53. Cell 112, 181-192 (2003)

(2003)
FURTHER READING Vogt, P. K. Fortuitous

convergences: the beginnings of JUN. Nature Rev. Cancer 2, 465-469 (2002)

WEB SITE

Erwin Wagner's lab:

http://emb1.bcc.univie.ac.at/PhD/Research/IMP/

Wagner/wagner.htm

\section{IN BRIEF}

\section{CHECKPOINTS}

Chk1-deficient tumour cells are viable but exhibit multiple checkpoint and survival defects.

Zachos, G. et al. EMBO J. 22, 713-723 (2003)

Chk1 is thought to initiate a checkpoint response to aberrant DNA structures, but its further study in multicellular organisms has been inhibited by the embryonic lethality of Chk1-deficient cells. Gene targeting has facilitated its deletion in the avian B-lymphoma DT-40 cell line; cells remain viable but have a defective checkpoint response and are sensitive to ionizing radiation. Chk1 seems to promote the survival of tumour cells by inhibiting mitosis, so its inhibition could be a therapeutic strategy.

\section{GENE PROFILING}

Identification of Hodgkin and Reed-Sternberg cell-specific genes by gene expression profiling.

\section{Küppers, R. et al. J. Clin. Invest. 111, 529-537 (2003)}

The tumorigenic cells of Hodgkin lymphoma are the Hodgkin and Reed-Sternberg cells, but they have been difficult to characterize. They originate from germinal-centre B cells and gene profiling has been performed to compare their gene expression with that of other normal and malignant B-cell types. They were found to be different to the other B cells and most like EBV-transformed B cells. Expression of several genes was specifically aberrant and these might represent targets for diagnostics and therapeutics.

\section{TUMOUR PROGRESSION}

\section{$\mathrm{IL}-1$ is required for tumor invasiveness and angiogenesis.}

Voronov, E. et al. Proc. Natl Acad. Sci. USA 18 Feb 2003 (doi:10.1073/pnas.0437939100)

The role of IL-1 in tumorigenesis was assessed by transplanting different tumour types into Il- $1 \alpha$ - and Il-1 $\beta$-knockout mice. The authors found that melanoma, mammary adenocarcinoma and prostate tumours grew more slowly and were less likely to metastasize in the knockout mice than in controls. Il- $1 \alpha$ and Il- $1 \beta$ were shown to contribute to tumour angiogenesis, but the role of Il-1 $\beta$ was more evident in these processes. The authors suggest that IL-1 antagonists might be effective anticancer therapeutics.

\section{DRUG TARGETING}

\section{Integrin-mediated targeting of drug delivery to} irradiated tumor blood vessels.

Hallahan, D. et al. Cancer Cell 3, 63-74 (2003)

Treatment of cancers with ionizing radiation induces expression of neoantigens, and these proteins present a tumour-associated target for therapy. Post-translational modification of integrins, including the fibrinogen receptor $\alpha 2 \mathrm{~b} \beta 3$ integrin, was induced by radiation and led to accumulation of these proteins within the tumour vasculature. Targeting irradiated tumours with fibrinogen-conjugated nanoparticles led to destruction of the vasculature and consequent tumour regression. 